\title{
QUASISYMMETRIC EMBEDDINGS OF A CLOSED BALL INEXTENSIBLE IN NEIGHBOURHOODS OF ANY BOUNDARY POINTS
}

\author{
Boris N. Apanasov
}

\section{Introduction}

The problem of the extension of a quasiconformal or quasisymmetric embedding $f: X \rightarrow R^{n}, X \subset R^{n}$, onto a large domain $Y \supset X$ is well known. A quasisymmetric embedding here is an embedding $f$ such that there is a homeomorphism $c:[0, \infty) \rightarrow[0, \infty)$ with the property

$$
|f(y)-f(x)| \leq c(r) \cdot|f(z)-f(x)|
$$

for all $x, y, z \in X$ such that $|y-x| \leq r|z-x|$. Then

$$
|f(y)-f(x)| \geq c^{\prime}(r) \cdot|f(z)-f(x)|
$$

if $|y-x| \geq r|z-x|$ and $c^{\prime}(r)=c\left(r^{-1}\right)^{-1}, c^{\prime}(0)=0$. Quasisymmetric embeddings are the natural generalization of quasiconformal mappings; see, for instance, [18]. In particular, a homeomorphism $R^{n}$ is quasiconformal if and only if it is quasisymmetric.

We are interested in the case where $X=\bar{B} \subset R^{n}$ is a closed ball. The fact that every quasisymmetric embedding of a closed disc $\bar{B}^{2} \rightarrow R^{2}$ is extended up to a quasiconformal automorphism of $\bar{R}^{2}$ (see $[9,18]$ ) is the well-known corollary of the $M$-condition of Ahlfors [1, Chapter IV].

A new phenomenon emerges with the growth of dimension. Namely, there appear quasisymmetric (even bi-Lipschitz) embeddings of a closed three-dimensional ball $\bar{B}^{3} \hookrightarrow \mathbf{R}^{3}$ which cannot be extended to an embedding of an open neighbourhood $U, \bar{B}^{3} \subset U \subset \mathbf{R}^{3}$. After this paper was completed, Jussi Väisälä pointed out to me that this phenomenon was discovered for the first time by F.W. Gehring [11], who constructed such an embedding with two singular points on the boundary of the ball (see detailed proof for this construction in the paper by G. Martin [15]).

The main result of this paper gives the construction of quasisymmetric embeddings of the closed ball $f: \bar{B}^{3} \hookrightarrow \mathbf{R}^{3}$ which are locally inextensible, i.e., inextensible to any neighbourhoods of any boundary points $x \in \partial B^{3}$. The most important 
part of our construction is the construction of some geometrically finite (see $[3,4]$ ) Kleinian group $G$ on the sphere $\mathbf{R}^{3}=\mathbf{R}^{3} \cup\{\infty\}$ (i.e., a discrete subgroup of the Möbius group $\mathrm{Möb}_{3}$ with the non-empty discontinuity set $O(G)=\overline{\mathbf{R}}^{3} \backslash L(G)$ ). This construction was also used by the author and A.V. Tetenov in the study of topological properties of hyperbolic 4-manifolds [7].

One can say that the embedding $f: B^{3} \rightarrow f\left(B^{3}\right)=O_{0}\left(O_{0}\right.$ being a component of $O(G)$ ) is obtained as resulting from quasiconformal deformation of action of some co-compact Fuchsian group on the ball-component $B^{3}$ of its discontinuity set. Here the property of local wildness of the embedding $\partial B^{3} \hookrightarrow \mathbf{R}^{3}$ is an obstruction to extending the embedding $f$ to any neighbourhood of any boundary point $x \in \partial B^{3}$.

One can interpret our construction as an infinite process of iterative knotting of a topological ball. It may seem possible to do this without using a group. However, we believe that it is not a realistic possibility (due to the necessity of having a wild knottedness of the ball on a dense subset of the boundary sphere).

I would like to thank Jussi Väisälä for his useful comments.

\section{Kleinian groups with a wildly knotted limit sphere}

We base our construction of geometrically finite Kleinian groups $G \subset \mathrm{Möb}_{3}$ whose limit set $L(G)$ is a wild sphere on an idea of periodicity of knotting used the author $[2,10]$ when constructing a wildly knotted limit curve $L(G) \subset R^{3}$.

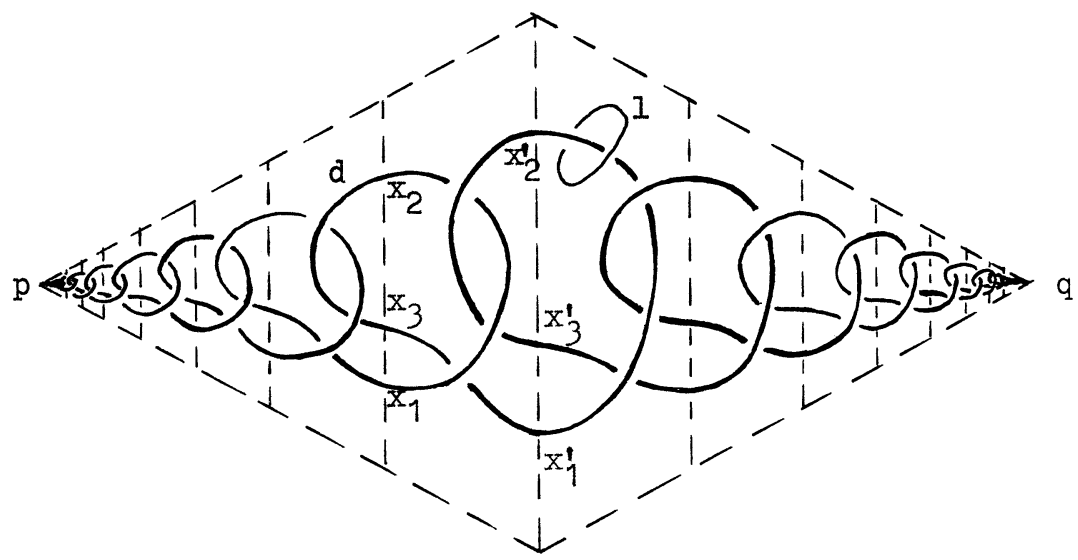

Figure 1.

Let us consider a wild Fox-Artin arc $d \subset R^{3}$ (knotted periodically) with endpoints $p$ and $q$; see Bing [9, Chapter IV.9]. By the word "periodically" we mean here that the arc $d$ is invariant for the action of some cyclic group generated 


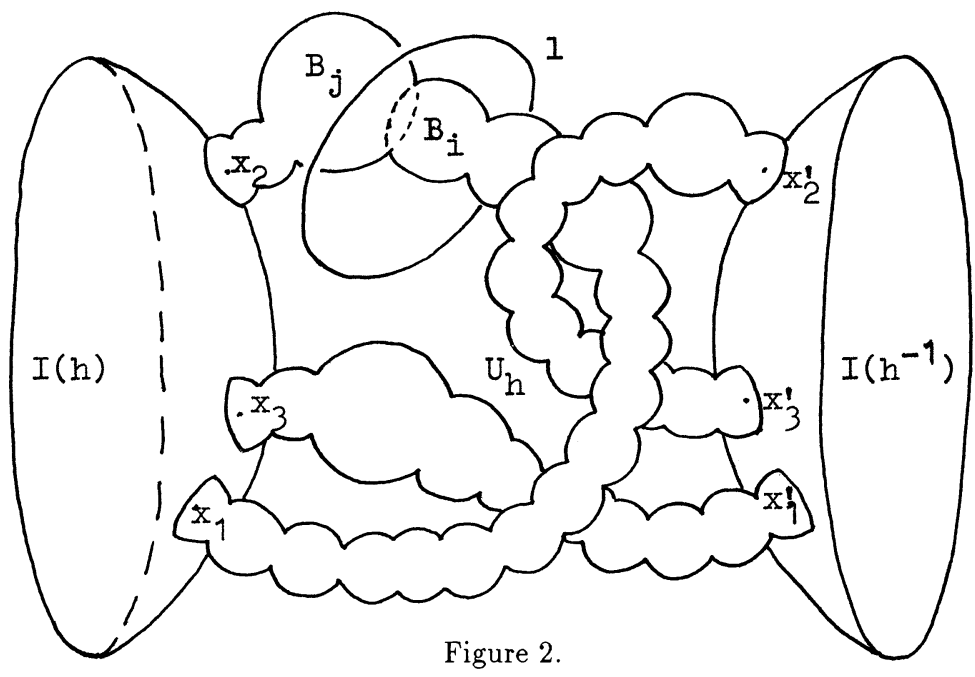

by a hyperbolic transformation $h \in \mathrm{Möb}_{3}$ such that $h(p)=p$ and $h(q)=q$, as seen in Figure 1.

Moreover, if $I(h)=\left\{x \in R^{3}:|D h(x)|=1\right\}$ and $I\left(h^{-1}\right)$ are the isometric spheres of $h$ and $h^{-1}, \quad h(\operatorname{ext} I(h))=\operatorname{int} I\left(h^{-1}\right)$, then $I(h) \cap d=\left\{x_{1}, x_{2}, x_{3}\right\}$ and $I\left(h^{-1} \cap d\right)=\left\{x_{1}^{\prime}, x_{2}^{\prime}, x_{3}^{\prime}\right\}$, where $h\left(x_{i}\right)=x_{i}^{\prime}$ and these points $x_{i}$ and $x_{i}^{\prime}$ are placed on $d$ in the following order:

$$
x_{1}, x_{2}, x_{3}, x_{1}^{\prime}, x_{2}^{\prime}, x_{3}^{\prime} .
$$

The intersection $d_{h}$ of the arc $d$ and ext $I(h) \cap \operatorname{ext} I\left(H^{-1}\right)$ consists of three arcs $\left(x_{1}, x_{2}\right),\left(x_{3}, x_{1}^{\prime}\right),\left(x_{2}^{\prime}, x_{3}^{\prime}\right)$ and forms the knotted period of $d$ on Figure 1.

Now take a neighbourhood $U_{h}$ of the three arcs of $d_{h}$ in the exterior of $I(h)$ and $I\left(h^{-1}\right)$ consisting of three disjoint tubes. For our further needs we can form it by the union of a finite number of consequently overlapping balls $B_{k}$. Here, according to the periodicity of $d$ it is necessary that $h\left(B_{k} \cap I(h)\right)=B_{j} \cap I\left(h^{-1}\right)$ if $x \in B_{k} \cap I(h)$ and $x_{i}^{\prime}=h\left(x_{i}\right) \in B_{j} \cap I\left(h^{-1}\right)$. It

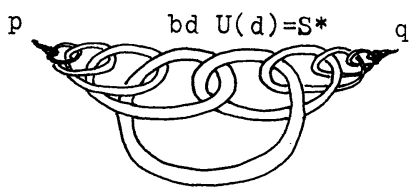
is easy to see that the closure of the spherical annuli $X_{k}=\mathrm{bd} B_{k} \cap \operatorname{ext} I(h) \cap \operatorname{ext} I\left(h^{-1}\right) \cap_{j \neq k}$ ext $B_{j}$ (see Figure 2) and their $h^{m}$-images, $m \in Z$, yields the fattening boundary bd $U(d)=\cup_{m, k} h^{m}\left(X_{k}\right) \cup\{p, q\}$ of the arc $d$ which is a wild Fox-Artin sphere $S^{*}$ in $R^{3}$; see Figure 3 .

Now we will form a finite family $C$ of spheres $S_{j}, j \in J$, with the following properties:

1. The union of the annuli $X_{k}$ is covered by the interiors of $S_{j}$.

2. For each $k, j$ either $B_{k} \cap S_{j} \neq \emptyset$ or $S_{j}$ is orthogonal to the sphere bd $B_{k}$; this also holds for $I(h)$ and $I\left(h^{-1}\right)$ taken instead of bd $B_{k}$. 
3. If $S_{i} \cap S_{j} \neq \emptyset$, the dihedral angle (exterior, i.e., containing $\infty$ ) between them equals $\pi / m$ for some integer $m$.

4. If $S_{i} \cap S_{j} \neq \emptyset$, there exists a common annulus $X_{k}$ for which $X_{k} \cap S_{i} \neq \emptyset$ and $X_{k} \cap S_{j} \neq \emptyset$.

5. There is a one-to-one correspondence between spheres $S_{j} \in C$ crossing $I(h)$, and spheres $S_{j} \in C$ crossing $I\left(h^{-1}\right)$ so that $S_{j}^{\prime}=h\left(S_{j}\right)$.

In other words, we will form a finite "bubble cover" of bd $U_{h}$ with good angles between the bubbles and right angles between the bubbles and spheres bd $B_{k}$, and respecting the periodicity. One can easily see that the freedom of choice of the balls $B_{k}$ (as well as the arc $d$ and the transformation $h$ ) permits us to vary conformal moduli of spherical annuli $X_{k}$ and thus obtain the sought-for family $C$.

However, taking into account the Andreev-Thurston rigidity of the circular covering of the sphere $\bar{R}^{2}$ (which is connected with the rigidity of hyperbolic space-forms and compact hyperbolic polyhedra; see [19, Chapter 13]), we do not restrict ourselves by the above arguments of existence of the family $C$, and we give its concrete construction for the chosen type of a Fox-Artin arc.

It will be obvious that our method is applicable for Fox-Artin arcs of any type.

\section{Construction of the special spherical covering $C$}

Let us consider a right prism $P$ in $R^{3}$ with the height 17 , whose base is a polygon coinciding with a union of 55 equal regular hexagons with unit sides and distinct interiors. These hexagons cover the regular triangle with the side $9 \sqrt{3}$ whose vertices are the centres of extreme hexagons; see Figure 4. Let us enumerate all the hexagons line by line so that the three extreme ones have numbers 1,10 and 55, and the central one has the number 31 . Divide the prism $P$ into $(55 \times 17)$ small hexagonal prisms $P(k, n)$ of unit height enumerated by pairs $(k, n)$, where $k, 1 \leq$ $k \leq 17$, is the "floor" of $P(k, n)$ and $n, 1 \leq n \leq 55$, is the number of hexagonal projection of $P(k, n)$ in the base.

Now let us associate with the three tubes, making up the neighbourhood $U_{h}$ of the knotting period $d_{h}=\left(x_{1}, x_{2}\right) \cup\left(x_{2}^{\prime}, x_{3}^{\prime}\right) \cup\left(x_{3}, x_{1}^{\prime}\right)$, three disjoint domains 
$=$ "broken tubes" $D\left(x_{1}, x_{2}\right), D\left(x_{2}^{\prime}, x_{3}^{\prime}\right)$ and $D\left(x_{3}, x_{1}^{\prime}\right)$ obtained by the union of small prisms $P(k, n)$ with the number-pairs $(k, n)$ from the following sets:

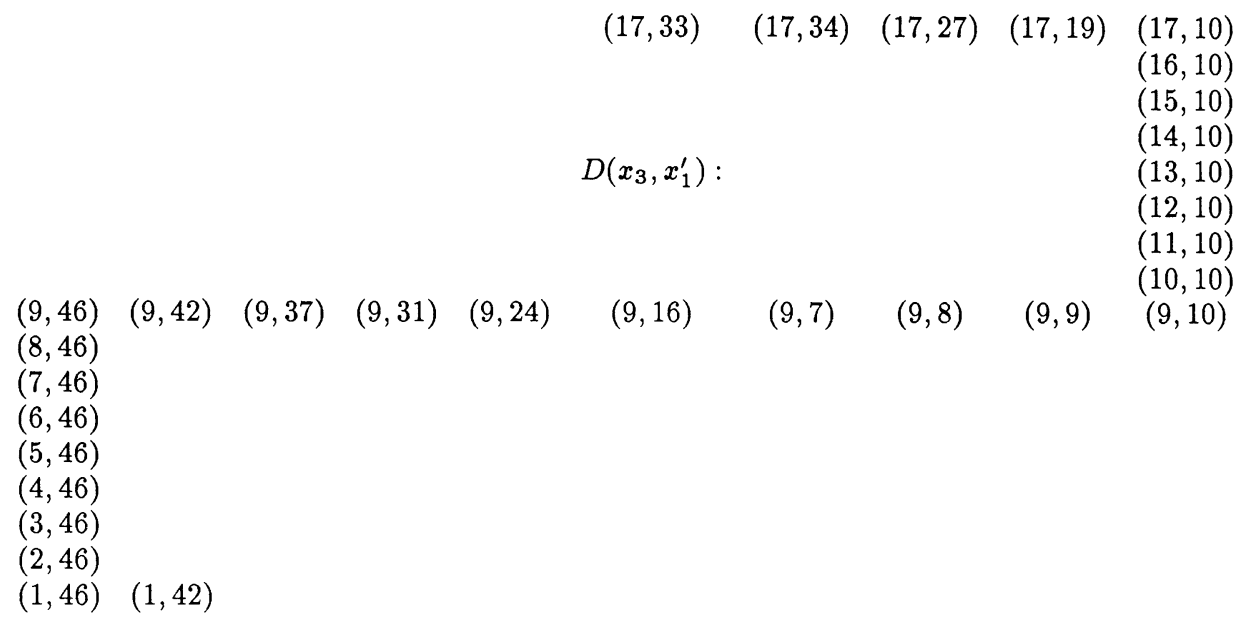

$$
\begin{aligned}
& \begin{array}{llllll}
(12,28) & (12,29) \quad(12,30) \quad(12,31) \quad(12,32) \quad(12,33) \quad(12,34)
\end{array} \\
& (11,28) \quad(11,34) \\
& (10,28) \quad(10,34) \\
& (9,28) \quad D\left(x_{1}, x_{2}\right): \quad(9,34) \\
& (8,28) \quad(8,34) \\
& (7,28) \quad(7,34) \\
& (6,28) \quad(6,20) \quad(6,11) \quad(6,1) \quad(6,34) \\
& (5,1) \quad(5,34) \\
& (4,1) \quad(4,34) \\
& (3,1) \quad(3,34) \\
& (2,1) \quad(2,34)
\end{aligned}
$$

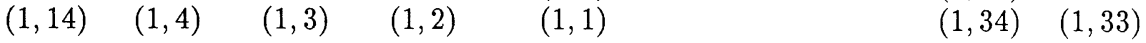

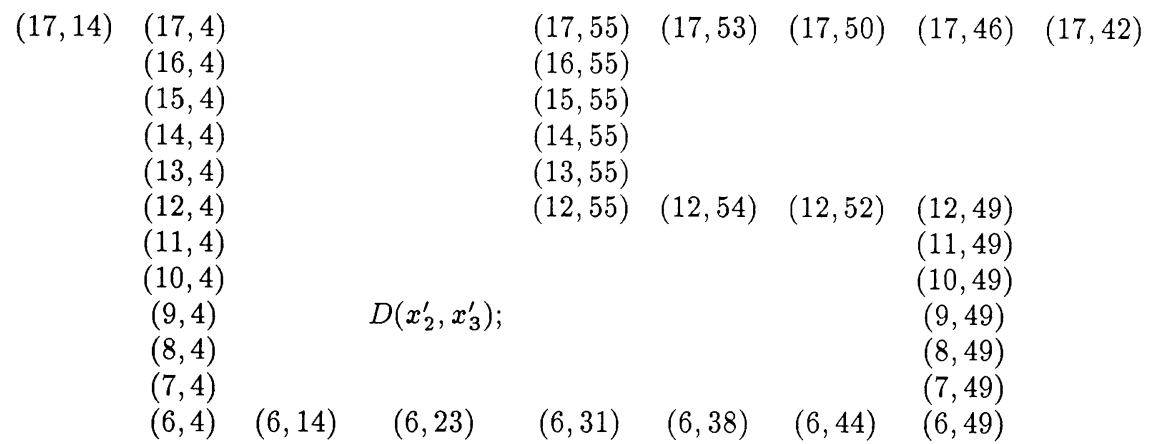


Let now $S_{i}$ be the spheres of radii $\sqrt{3} / 3$ with the centres in vertices of prisms $P(k, n)$ forming the domains $D(*, *)$. If such spheres $S_{i}$ and $S_{j}$ intersect, their centres are the adjacent vertices of some prism $P(k, n) \subset P$ and their angle of intersection is $\pi / 3$.

Denote by $B(k, n)$ the ball with the centre in the centre of the prism $P(k, n)$ and of radius $\sqrt{11 / 12}$. Its boundary sphere $S(k, n)$ is orthogonal to each of the spheres $S_{i}$ whose centres are the vertices of $P(k, n)$. After that we may assume that the balls $B_{k}$ whose union is the three components of $U_{h}$ are the balls $B(i, n)$ corresponding to the prisms $P(i, n)$ from the domains $D(* . *)$.

For the isometric spheres $I(h)$ and $I\left(h^{-1}\right)$ take the spheres $S^{2}\left(z_{1}, \sqrt{83 / 12}\right)$ and $S^{2}\left(z_{17}, \sqrt{83 / 12}\right)$, respectively, of the radii $\sqrt{83 / 12}$ and with the centres at the points $z_{1}$ and $z_{17}$ which are the centres of the 1 st and the 17 th floors, i.e., the centres of the prisms $P(1,31)$ and $P(17,31)$. Here, the radius of these spheres is such that they only intersect the spheres $S(1,14), S(1,33), S(1,42)$, and $S(17,14)$, $S(17,33), S(17,42)$, respectively, and they are orthogonal to the spheres $S_{i}$ with the centres at vertices of the prisms $P(1, n)$ and $P(17, n)$ which are nearest to $z_{1}$ and $z_{17}$, respectively.

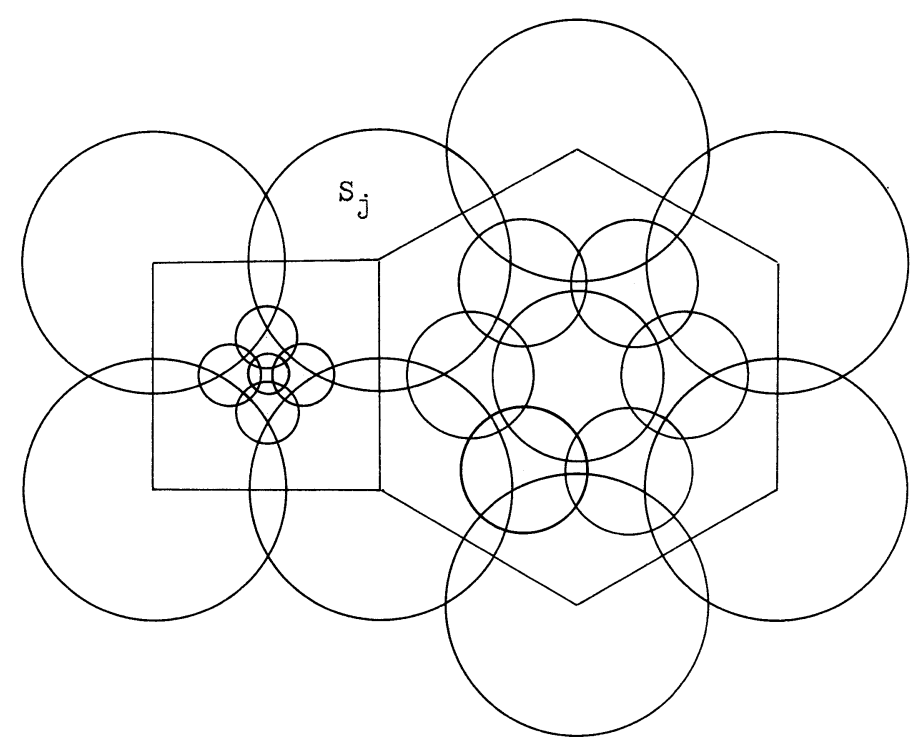

Figure 5 .

Note that the interiors of the spheres $S_{i}$ with the centres at the vertices of the prisms $P(k, n)$ do not cover the entire boundary bd $U_{h}$, i.e., not all the spherical annuli $X_{i} \subset S(k, n)$. The hexagonal and quadrangular domains on these annuli, corresponding to sides of the prisms $P(k, n)$, are still uncovered. Let us cover 
each hexagonal domain on $X_{i} \subset S(k, n)$ with the union of the interiors of seven spheres orthogonal to the sphere $S(k, n)$. Six of them have equal radii, they are orthogonal to the spheres $S_{i}$ and so intersect at the angles $\pi / 3$. The seventh sphere intersects six others at the right angles and does not intersect the spheres $S_{i}$ with the centres at vertices of $P(k, n)$; see Figure 5 .

We cover each quadrangular domain on $X_{i} \subset S(k, n)$ by interiors of five spheres orthogonal to the sphere $S(k, n)$. Four of these spheres have equal radii, they are orthogonal to the spheres $S_{j}$ and so intersect at the angles $\pi / 3$. The fifth sphere intersects four others at right angles and does not intersect the spheres $S_{j}$; see Figure 5.

Direct calculation shows that the covering by the spheres of the boundary bd $U_{h}$ thus obtained has all the properties 1-5 of the family $C$. This completes the construction of the family $C$.

Finally, let us note that properties 2 and 4 of the family $C$ (where 2 in the case $S_{k} \cap B_{i} \neq \emptyset$ and $S_{k} \cap B_{j} \neq \emptyset$ means that $S_{k}$ is orthogonal to the circle $b_{i j}=\operatorname{bd} B_{i} \cap \mathrm{bd} B_{j}$ ) allow us to "bend" cylindrical surfaces forming bd $U_{h}$, and thus to bend the whole surface $S^{*}$ along the circles bounding the spherical annuli $h^{m}\left(X_{i}\right)$ without changing dihedral angles between the spheres of our covering $\left(h^{m}(C)\right)$ of the surface $S^{*}$.

Denote by $G_{1}, G_{2}$ and $G_{3}$ three Möbius groups generated by reflections with respect to spheres $S_{j}$ whose interiors intersect the domains $D\left(x_{1}, x_{2}\right), D\left(x_{2}^{\prime}, x_{3}^{\prime}\right)$ and $D\left(x_{3}, x_{1}^{\prime}\right)$, respectively. These groups are discrete due to the property 3 of the family $C$ and to the Poincaré-Aleksandrov theorem on fundamental polyhedra (see [5]). The application in $R^{3}$ of Maskit's first combination theorem (see [4, Theorem 4.2]) shows that the groups $G_{k}$ are amalgamed free products:

$$
G_{k}=G_{k 1} *_{H_{12}} G_{k 2} *_{H_{23}} G_{k 3} *_{H_{34}} \cdots * G_{k m_{k}}, \quad k=1,2,3,
$$

where, for all $i$, groups $G_{k i}$ are generated by reflections with respect to spheres of the family $C$ which intersect the ball $B_{i}, B_{i} \cap D(*, *) \neq \emptyset$, and every amalgamsubgroup $H_{i j}$ is generated by reflections with respect to spheres from $C$ whose interiors cover the circle $b_{i j}=\mathrm{bd} B_{i}$ bd $B_{j}$.

Then, using non-intersection of spheres $S_{j}$ covering distinct domains $D(*, *)$, i.e, non-intersection of the groups $G_{1}, G_{2}$ and $G_{3}$, we see from Klein's combination theorem ([4, Theorem 4.1]) that the discrete (due to property 3 of $C$ ) Möbius group $G_{0}$ generated by reflections with respect to the spheres of $C$ is the free product of the groups $G_{k}: G_{0}=G_{1} * G_{2} * G_{3}$.

Denote by $H_{i}$ (respectively $H_{i}^{\prime}$ ),i=1,2,3, the subgroups in $G_{0}$ generated by reflections in spheres of $C$, covering the circles $I(h) \cap B_{j}$ (respectively $I\left(h^{-1}\right) \cap B_{j^{\prime}}$ ) inside which the points $x_{i}$ are (respectively the points $x_{i}^{\prime}$ ). And let

$$
H=H_{1} * H_{2} * H_{3}, \quad H^{\prime}=H_{1}^{\prime} * H_{2}^{\prime} * H_{3}^{\prime} .
$$


Then the group $G$ generated by the group $G_{0}$ and by the hyperbolic element $h, h(\operatorname{ext} I(h))=\operatorname{int} I\left(h^{-1}\right)$, is a discrete group obtained by HNN-extension of the group $G_{0}$ by means of the element $h$ conjugating the subgroups $H$ and $H^{\prime}$ of the group $G_{0}$. This fact follows from Maskit's second combination theorem ([4, Theorem 4.5]), since the closed balls $\mathrm{cl}(\operatorname{int} I(h))$ and $\mathrm{cl}\left(\operatorname{int} I\left(h^{-1}\right)\right)$ are precisely invariant in the group $G_{0}$ with respect to the subgroups $H$ and $H^{\prime}$, respectively. Thus, we have the following copresentation of the group $G$ :

$$
G=\left\langle G_{1} * G_{2} * G_{3}, h: h H_{i} h^{-1}=H_{i}^{\prime}, \quad i=1,2,3\right\rangle,
$$

having, as a fundamental polyhedron, the following finite-sided spherical polyhedron $F$ which has four connection components: an unbounded one $F_{\infty}$ and three bounded ones $F_{i}$,

$$
F=\bigcap\left\{\operatorname{ext} S_{j}: S_{j} \in C\right\} \cap \operatorname{ext} I(h) \cap \operatorname{ext} I\left(h^{-1}\right) .
$$

Note that if we extend the spheres $I(h), I\left(h^{-1}\right)$ and $S_{j} \in C$ up to hyperbolic 3 -planes in $H^{4}=R_{+}^{4}$, we obtain a finite-sided convex polyhedron $F^{4} \subset H^{4}$ which is similar to the polyhedron (2.2) and fundamental for the action of $G$ in $H^{4}$. This shows the geometric finiteness of the group $G$.

If in $G$, instead of the generators contained in the group $G_{1}$, we take their conjugations by the element $h$ (i.e., the elements from the group $h G_{1} h^{-1}$ ), we obtain (by means of Tietze's transformations; see [14, Section 2.2]) that copresentation (2.1) is rewritten in new generators in the following form:

$$
G=\left\langle G_{2} *_{H_{2}^{\prime}} h G_{1} h^{-1} *_{H_{1}^{\prime}} G_{3}, h: h H_{3} h^{-1}=H_{3}^{\prime}\right\rangle .
$$

In other words, the copresentation (2.3) implies that the group $G$ is the HNNextension of the group $G_{0}^{*}$ by means of the element $h$, where $G *_{0}$ is generated by reflections in spheres of the family $C^{*}=C_{2} \cup C_{3} \cup h\left(C_{1}\right)$. Here, the subfamily $C_{i} \subset C, i=1,2,3$, consists of spheres $S_{j} \in C$ intersecting the $i$ th domain $D(*, *)$ (related to the group $G_{i}$ ).

It can easily be seen that, instead of the polyhedron $F$ from $(2.2)$ as a fundamental polyhedron of the group $G$, one may take the polyhedron

$$
F^{*}=F_{\infty} \cup F_{2} \cup F_{3} \cup h\left(F_{1}\right)
$$

consisting of the unbounded component $F_{\infty}$ and of the connected bounded one $F_{0}=F_{2} \cup F_{3} \cup h\left(F_{1}\right)$ containing the segment $\left(x_{3}, x_{3}^{\prime}\right)$ of the arc $d$.

From the construction of the group $G$ we see ([4, Lemma 3.7]) that the domains

$$
O_{0}=\bigcup\left\{g\left(F_{0}\right): g \in G\right\} \quad \text { and } \quad O_{1}=\bigcup\left\{g\left(F_{\infty}\right): g \in G\right\}
$$

are $G$-invariant components of the discontinuity set $O(g)$ and, due to the fundamentality of $F^{*}$ for the group $G, O(G)=O_{0} \cup O_{1}$.

Now it remains to be seen that the following statement is valid: 
Lemma 2.1. The limit set $L(G)$ of the geometrically finite group $G \subset$ $\mathrm{Möb}_{3}$, constructed above, is a two-dimensional sphere wildly embedded in $R^{3}$ and dividing the sphere $\bar{R}^{3}$ into two $G$-invariant components, one of them being a quasiconformal ball.

The proof of this fact will be given in the next section.

\section{Construction of a wild quasisymmetric embedding of a ball}

Our goal is to contruct a homeomorphism $\hat{f}: \bar{O}_{0}=O_{0} \cup L(G) \rightarrow \bar{B}$ of closure of the connection component $O_{0} \subset O(G)$ from (2.5) upon a closed ball $\bar{B} \subset$ $R^{3}$. This homeomorphism should be quasiconformal in $O_{0}$, quasisymmetric on its boundary and compatible with the group $G$ of Section 2 . Hence, in particular, the fact from Lemma 2.1 follows that the limit set $L(G)$ is a 2 -sphere.

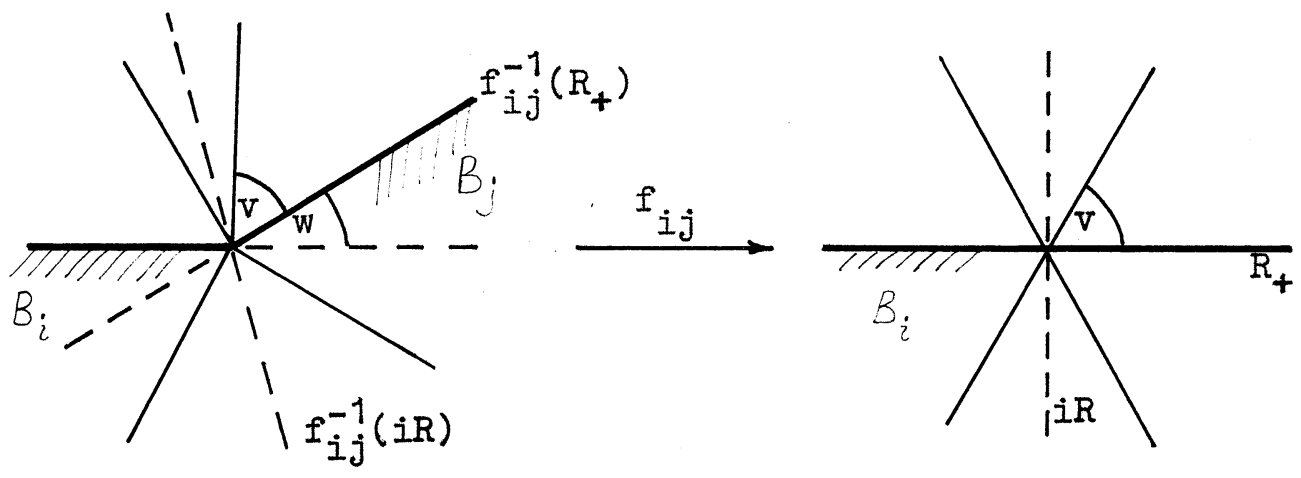

Figure 6 .

Let $B_{i}$ and $B_{j}$ be any two adjacent intersecting balls from our construction in Section 2 (or $h$-images of such balls), and let $b_{i j}=\mathrm{bd} B_{i} \cap B_{j}$. Then there exists a quasiconformal homeomorphism $f_{i j}$ transferring the domain $B_{i} \cup B_{j}$ to the ball $B_{i}$ and conformal in a neighbourhood of the spherical discs $\left(\mathrm{bd} B_{i}\right) \backslash \bar{B}_{j}$ and $\left(\mathrm{bd} B_{j}\right) \backslash \bar{B}_{i}$. Namely, let $B_{i}$ and $B_{j}$ be just half-spaces, the planes (bd $B_{i}$ ) and $\left(\mathrm{bd} B_{j}\right)$ containing the origin and intersecting at the angle $w, 0<w<\pi$, on the third coordinate axis $b_{i j}=\{(0,0, t): t \in R\}$. Let us also have the natural complex structure $\left(z=x_{1}+i x_{2}\right)$ in the plane $R^{2}=\left\{x \in R^{3}: x_{3}=0\right\}$ and let $v$ be a fixed number such that

$$
0<v<\pi / 2, \quad 0<w<\pi-2 v .
$$

Then the quasiconformal homeomorphism $f_{i j}$ is described by its behaviour (pro- 
jection) on the plane $\mathbf{C}=R^{2}$ where

$$
f_{i j}^{-1}(z)= \begin{cases}z & \text { if }|\arg z| \geq \pi-v, \\ z \cdot \exp (i w) & \text { if }|\arg z| \leq v, \\ z \cdot \exp (i w(1-(\arg (z)-v) /(\pi-2 v))) & \text { if } v<\arg z<\pi-v \\ z \cdot \exp (i w(1+(\arg (z)+v) /(\pi-2 v))) & \text { if } v-\pi<\arg z<-v\end{cases}
$$

and similar homeomorphisms transfer isometric spheres of $H$ to spheres orthogonal to the boundary of $B$.

Considering the composition of the finite number of all such quasiconformal homeomorphisms $f_{i j}$ of the (3.1) type, we obtain a quasiconformal homeomorphism $f_{0}$ of the polyhedron $F_{0}$ into a ball $B \subset R^{3}$ which transfers the sides of the polyhedron $F_{0}$ to the sides of some polyhedron $F_{0}^{\prime}=f\left(F_{0}\right)$ lying on spheres orthogonal to the boundary sphere bd $B$. Here, due to the conformality property of the homeomorphisms $f_{i j}$ in the neighbourhoods of the above-mentioned discs and due to properties 2 and 4 of the family $C$, all dihedral angles $W$ and $W^{\prime}=f(W)$ of the polyhedra $F_{0}$ and $F_{0}^{\prime}$ are equal. This proves the following important fact:

Let $G^{\prime}$ be a Möbius group generated by reflections in the sides of the polyhedron $F_{0}^{\prime}$ (except for the sides on $f_{0}(I(h))$ and on $f_{0}\left(I\left(h^{-1}\right)\right)$ and by a hyperbolic transformation $h_{0} \in \mathrm{Möb}_{3}$ which transfers $f_{0}(I(h))$ to $f_{0}\left(I\left(h^{-1}\right)\right)$. Then, applying Maskit's 1 st and 2nd combination theorem ([4, Theorem 4.3 and 4.5]), we find that the group $G^{\prime}$ is the HNN-extension of the group $G_{0}^{\prime} \subset G^{\prime}$ generated by all reflections in the sides of $F_{0}^{\prime}$ by means of the element $h_{0}$. This subgroup $G_{0}^{\prime}$ (as the group $G_{0} \subset G$ ) is the free product of its subgroups $G_{i}^{\prime}, G_{j}^{\prime}$ with amalgamated subgroups $G_{i}^{\prime} \cap G_{j}^{\prime}$. Thus, the information about the dihedral angles $W^{\prime}=f_{0}(W)$ of the polyhedron $F_{0}^{\prime}$, the property 3 of the family $C$ and the copresentation 2.3 of the group $G$ prove that $G^{\prime} \subset \mathrm{Möb}_{3}$ is discrete and isomorphic to the group $G$ Möbius group in the ball $B$ with a compact quotient $B / G^{\prime}$.

Moreover, extending the mapping $f_{0}: F_{0} \rightarrow F_{0}^{\prime}$ compatible with the actions of the groups $G$ and $G^{\prime}$ upon images $G\left(F_{0}\right)$, i.e., upon the whole component $O_{0} \subset O(G)$, we obtain a quasiconformal mapping $f: O_{0} \rightarrow B$ conjugating the actions of the groups $G$ and $G^{\prime}$ :

$$
G^{\prime}=f G f^{-1}
$$

Now it remains to extend the mappings $f$ up to a homeomorphism $\hat{f}: \bar{O}_{0} \rightarrow \bar{B}$ on closed domains. The mapping $\hat{f}$ is obtained by the usual extension by continuity. This fact follows from Tukia's theorem ([17, Theorem 3.3 and Lemma 3.7]) about isomorphisms of geometrically finite Kleinian groups. We formulate this result as

Theorem 3.1 (Tukia). Let $G$ and $G^{\prime}$ be geometrically finite Kleinian groups in $\bar{R}^{n}$ and let $I: G \rightarrow G^{\prime}$ be an isomorphism preserved types of elements. Then 
there exists a homeomorphism $f_{I}: L(G) \rightarrow L\left(G^{\prime}\right)$ of the limit sets, induced by $I$ and unique in the case of a non-elementary group $G$. Here, $f_{I} \mid L(G) \cap R^{n}$ is quasi-symmetric if $\infty \notin L(G) \cup L\left(G^{\prime}\right)$ or $f_{I}(\infty)=\infty$.

Moreover, let $X \subset O(G)$ be a $G$-invariant set such that $X / G$ is compact, and let $f: X \rightarrow O\left(G^{\prime}\right)$ be a continuous mapping inducing the isomorphism $I$. Then $f$ and $f_{I}$ yield a continuous mapping $\hat{f}: L(G) \cup X \rightarrow \bar{R}$, which is an embedding if $f$ is an embedding.

To apply this theorem directly to our situation, it suffices to note that the geometrically finite group $G$ from Section 2 contains no parabolic elements. Therefore, for the set $X=O_{0}$, its quotient $X / G$ is a compact orbifold.-See [3, 4]. Thus, we have constructed the sought-for homeomorphism

$$
\hat{f}: O_{0} \cup L(G)=\bar{O}_{0} \rightarrow \bar{B} \subset R^{3},
$$

which is quasiconformal in $O_{0}$ and quasisymmetric on its boundary bd $O_{0}=L(G)$. Moreover, from Corollary 3.2 of Aseev's work [8] we immediately see that the homeomorphism $\hat{f}$ is quasisymmetric on the whole closed domain $\bar{O}_{0}$.

Now, to complete the proof of Lemma 2.1, it suffices to prove that the topological sphere $L(G)=\hat{f}^{-1}(\mathrm{bd} B)$ is wildly embedded in $R^{3}$. This follows from Alexander's theorem about the non-knottedness of any polyhedral 2 -sphere in $R^{3}$ and from the fact that the second component $O_{1}$ of the complement $\bar{R}^{3} \backslash L(G)=O(G)$ has the nontrivial fundamental group $\pi_{1}\left(O_{1}\right) \neq 0$.

Indeed, consider a nontrivial (in $F_{\infty}$ ) loop $l$ in the unbounded component $F_{\infty} \subset O_{1}$ of the fundamental polyhedron $F^{*}$ from (2.4) of the group $G$; see Figure 2. Suppose that $l$ is contractible in $O_{1}=G\left(F_{\infty}\right)$. Then, due to Dehn's lemma (see, for reference [4, Theorem 8.4]), there exists a topological disc $D \subset O_{1}$, bd $D=l$. Due to the compactness of the disc $D$, it is covered by a finite number of polyhedra $g_{i}\left(F_{\infty}\right), g_{i} \in G$. At the same time, due to the nontriviality of the loop $l$ in the complement $R^{3} \backslash d$ (see [10, Chapter IV.9]), we have $D \cap d \neq \emptyset$. Therefore, there exists $g_{0} \in G$ such that $g_{0}\left(F_{\infty}\right) \cap d \neq \emptyset$. The obtained contradiction with $d \subset O_{0} \cup(p, q)$ completes the proof of the nontriviality of $\pi_{1}\left(O_{1}\right)$ and thus completes the proof of Lemma 2.1 .

From Lemma 2.1 and from our construction of the mapping $\hat{f}$ from (3.2) it follows that $\hat{f}$ cannot be homeomorphically extended in any neighbourhood of the points $p$ and $q$ which are the wild knotting points of the sphere $L(G)=\operatorname{bd} O_{0}$. This conclusion may be essentially strengthened:

Theorem 3.2. The quasisymmetric embedding $\hat{f}^{-1}: \bar{B} \rightarrow \bar{O}_{0} \subset R^{3}$ of a closed ball $\bar{B}$ in $R^{3}$ (which is quasiconformal inside $B$ ) cannot be extended up to an embedding of any neighbourhood of any its boundary points $x \in \partial B$.

To prove this statement, it suffices to note that a set of points $z \in L(G)$, in whose neighbourhood the topological sphere $L(G)$ is wildly knotted, is a dense 
subset in $L(G)$. This follows from the density on $L(G)$ of the $G$-orbit $G(\{p, q\})$ of the arc ends $p$ and $q$, which are fixed points of the hyperbolic element $h \in G$ (see [12, Lemma 1]). Moreover, the points $g(p)$ and $g(q)$ of this $G$-orbit $\{g(\{p, q\})$ : $g \in G\}$ are fixed for the hyperbolic elements $g h g^{-1}$.

In terms of the function theory of several complex variables, the embedding from Theorem 3.2 may be called a quasisymmetric obstruction for the closed ball $\bar{B}$.

One may ask a question about the properties of closed domains admitting quasisymmetric obstructions. Obviously, a class of such domains contains closed domains which are quasisymmetric images of 3 -dimensional ball. Using the author's methods of [6] one can construct contractible domains in $R^{3}$ which are not quasisymmetric images of a ball, but have quasisymmetric obstructions.

Besides, three circumstances should be noted.

Firstly, though the method used in the paper is global, the property of a domain to have quasisymmetric obstruction is likely to be a local property of its boundary.

Secondly, the domain $O_{0} \subset O(G) \subset R^{3}$ with quasisymmetric obstruction constructed in the paper is, as can easily be seen from the construction, John's domain (see Reshetnjak [16]), i.e, there exists $R>0$ such that any point $x \in O_{0}$ may be connected in $O_{0}$ with a fixed point $a \in O_{0}$ by a rectifiable curve $x(s)$, $0 \leq s \leq l$, (the parameter $s$ being the length of the arc), for which $x(0)=x$, $x(l)=a$ and the inequality

$$
\operatorname{Dist}_{\mathrm{Eucl}}\left(x(s), \operatorname{bd} O_{0}\right) \geq s R / l
$$

is valid for every $s \in(0, l)$.

Thirdly, the quasisymmetric embedding $\bar{B}^{3} \hookrightarrow R^{3}$ constructed in the paper probably cannot be a limit of a sequence of quasiconformal self-maps of $R^{3}$ compatible with the action of the discrete group $G^{\prime}$ in $B^{3}$; compare [20, Chapter 5].

\section{References}

[1] Ahlfors, L.V.: Lectures on quasiconformal mappings. - D. Van Nostrand Company; Princeton, 1966.

[2] Apanasov, B.N.: Kleinian groups, Teichmüller space, and Mostow's rigidity theorem. Sibirsk. Mat. Zh. 21:4, 1980, 3-15. Siberian Math. J. 21, 1980, 483-491 (Russian).

[3] Apanasov, B.N.: Geomerical finite hyperbolic structures on manifolds. - Ann. of Glob. Analysis and Geometry 1:3, 1983, 1-22.

[4] Apanasov, B.N.: Discrete transformation groups and structures on manifolds. - "Nauka", Novosibirsk, 1983 (Russian).

[5] Apanasov, B.N.: The effect of dimension four in Aleksandrov's problem of filling a spaçe by polyhedra. - Ann. of Glob. Analysis and Geometry 4:2, 1986, 243-261.

[6] Apanasov, B.N.: Deformations of conformal structures on manifolds related to totally geodesic submanifolds. - Dokl. Akad. Nauk SSSR 293, 1987, 11-14. Soviet Math. Dokl. 34, 1987 (Russian). 
[7] Apanasov, B.N., and A.V. Tetenov: Nontrivial cobordisms with geometrically finite hyperbolic structures. - J. of Diff. Geom. 28, 1988, 407-422.

[8] Aseev, V.V.: Normal families of topological embeddings. - Dinamika sploshnoi sredy, Inst. Fluid Dinamics, Novosibirsk, 76, 1986, 32-42 (Russian).

[9] Aseev, V.V., and I.V. Zhuravlev: On quasiconformal extension of planar homeomorphisms. - Isvestia Vis. Ucheb. Zav. Math. 9, 1986, 3-6 (Russian).

[10] Bing, R.H.: The geomteric topology of 3-manifolds. - Colloquium Publ. 40, American Mathematical Society, Providence, 1983.

[11] Genring, F.W.: Extension theorems for quasiconformal mappings in $n$-space. - Proceedings of the International Congress of Mathematicians 1966, Moscow, 1968, 313-318.

[12] Greenberg, L.: Discrete subgroups of the Lorentz group. - Math. Scand. 10, 1962, 85107.

[13] Krushkal', S.L., B.N. Apanasov, and N.A. Gusevskir: Kleinian groups and uniformization in examples and problems. - Transl. Math. Monographs 62, American Mathematical Society, Providence, 1986.

[14] Lyndon, R.C., and P.E. Schupp: Combinatorial group theory. - Ergebnisse Math. 89, Springer-Verlag, Berlin, 1977.

[15] Martin, G.E.: Quasiconformal and bi-Lipschitz homeomorphisms, uniform domains and the quasihyperbolic metric. - Trans. Amer. Math. Soc. 292, 1985, 169-191.

[16] Reshetnyak, YU.G.: Stability theorems in geometry and analysis. - "Nauka", Novosibirsk, 1982, 1-230.

[17] Tukia, P.: On isomorphisms of geomterically finite Möbius groups. - Inst. Hautes Etudes Sci. Publ. Math. 61, 1985, 171-214.

[18] TukiA, P., and J. VÄIs̈̈L̈̈: Quasisymmetric embeddings of metric spaces. - Ann. Acad. Sci. Fenn. Ser. A I Math. 5, 1980, 97-114.

[19] Thurston, W.P.: The geometry and topology of 3-manifolds. - Lecture notes, Princeton University, 1978/1980.

[20] Apanasov, B.N.: Deformations of conformal structures and Lobachevsky geometry. Math. Sci. Res. Inst. preprint 02723-89, Berkeley, California, February 1989.

Siberian Branch of the USSR Academy of Sciences

Institute of Mathematics

Novosibirsk

U.S.S.R. 630090

Received 3 November 1987 